\title{
Relationship between sleep quality and cardiovascular disease risk in Chinese post-menopausal women
}

\author{
Sek Ying Chair ${ }^{1}$, Qun Wang ${ }^{1 *}$, Ho Yu Cheng ${ }^{1}$, Sally Wai-Sze Lo ${ }^{1}$, Xiao Mei Li², Eliza Mi-Ling Wong ${ }^{1}$ \\ and Janet Wing-Hung Sit $^{1}$
}

\begin{abstract}
Background: Menopause is an inevitable stage affecting every middle-aged woman. China has a large and increasing group of post-menopausal women. Most post-menopausal women suffer from increased risks for cardiovascular diseases (CVD) and sleep problems. Previous studies have demonstrated the associations between sleep disorders and increased CVD risks in general population. The current study is to examine the relationship between sleep quality and CVD risks among Chinese post-menopausal women.

Methods: This study was a sub-study nested in a cross-sectional study that investigated the sleep quality of community-dwelling adults in Xian, Shaanxi Province, China. The Chinese version of the Pittsburgh Sleep Quality Index (PSQI) and the Framingham 10-year risk score (FRS) were used to measure sleep quality and CVD risk among 154 Chinese post-menopausal women. Multivariate regression and logistic regression were used to determine the association between sleep quality and CVD risk.

Results: The participants (age: $63.65 \pm 4.47$ years) experienced poor sleep quality (mean score of global PSQI $=8.58$ ) and a 10-year risk of CVD of 12.54\%. The CVD risk was significantly associated with sleep duration $(\beta=-0.18, p=0.04)$ and sleep disturbance $(\beta=0.33, p<0.001$ ). Women with good sleep quality (PSQI $\leq 5)$ were less likely to be at high risk for CVD (FRS > 10\%) (odds ratio $=0.51, p=0.04$ ).

Conclusions: Poor sleep quality might increase the CVD risk in post-menopausal women. Interventions to promote the cardiovascular health of Chinese post-menopausal women may need to include sleep promotion strategies.
\end{abstract}

Keywords: Cardiovascular disease risk, Post-menopausal women, Sleep quality

\section{Background}

Menopause is a natural and an inevitable stage leading to old age that affects every middle-aged woman. Every day, about 6000 American women reach menopause [1]. In China, 120 million women, accounting for around $23 \%$ of the world's menopausal population, are experiencing the transition to menopause [2]. With the ageing population and rise in life expectancy worldwide, the stages of menopause and post-menopause will account for about one third of women's lives [1]. Moreover, the

\footnotetext{
* Correspondence: qunwang@cuhk.edu.hk

'The Nethersole School of Nursing, The Chinese University of Hong Kong, Esther Lee Building, The Chinese University of Hong Kong, Shatin, New Territories, Hong Kong

Full list of author information is available at the end of the article
}

number of post-menopausal women is also expected to increase. It is estimated that there will be 1.1 billion post-menopausal women in the world by 2025 [1]. Therefore, the health of this large population group is of high importance to public health.

Menopause is often accompanied by an increased risk for cardiovascular diseases (CVD) and sleep problems [3-5]. During the normal physiological process of menopause, the reduction in ovarian follicular function often leads to the depletion of estrogen, contributing to the development of a series of cardio-metabolic risks. These risks include central obesity, reduced glucose tolerance, increased blood pressure, abnormal levels of plasma lipids, and vascular inflammation [6], and as a consequence, substantially increasing the CVD risk and 
mortality in this group of women [7]. The hormonal changes that occur during menopause have also been reported to be correlated with various sleep problems [8]. The common complaints among post-menopausal women include difficulty in sleep onset, short sleep duration, and poor sleep quality [9]. It was reported that around $53.3 \%$ to $65.8 \%$ of post-menopausal women suffered from sleeplessness during their menopausal transition [10].

Numerous studies have demonstrated the associations between sleep disorders and increased CVD risk in general population [11]. An increased risk for CVD and coronary heart disease (CHD) incidents was found in individuals who had short sleep duration $(\leq 6 \mathrm{~h})$ and poor sleep quality [11]. Furthermore, in those who had sleepdisordered breathing (e.g., apnea and hypopnea), the relative risk for heart failure and stroke was 2.38 and 1.58, respectively [12]. However, limited studies examined the relationship of these health-related aspects particularly among post-menopausal women, and even fewer studies were conducted among Chinese post-menopausal women, the largest population group of this type in the world.

Previous studies among general populations usually assessed sleep quality by a single item, such as "How often is your sleep satisfactory?", "How many hours of sleep do you usually get?" or "How often do you experience insomnia?" [11]. Moreover, limited evidence was obtained from a comprehensive and validated assessment of sleep quality. Thus, the present study aimed to investigate sleep quality using a comprehensive and validated tool and to examine the relationship between sleep quality and the CVD risk among Chinese postmenopausal women.

\section{Methods}

\section{Study design and participants}

This study was a secondary data analysis of a crosssectional study conducted in Xi'an, Shaanxi Province, China. The original cohort consisted of 404 communitydwelling adults, whose sleep quality and its associated factors were examined [13]. The present study focused on the data of 154 post-menopausal women from the original cohort to evaluate their sleep quality and CVD risk. Post-menopause was defined as amenorrhea not attributable to other causes for at least 12 months [14]. The eligibility of the participants was assessed by their self-reported menstrual history.

\section{Measures}

Data were collected by a structured questionnaire that included the Pittsburgh Sleep Quality Index (PSQI), items on cardiovascular parameters, and socio-demographic information. All questionnaires were administered by one researcher through face-toface interviews.

\section{Pittsburgh sleep quality index}

Sleep quality was assessed by the Chinese-version of the PSQI (PSQI-C) [15]. The PSQI-C consists of 19 selfrated items related to sleep quality in the past month, of which seven component scores are summed to give a global score. The seven components include subjective sleep quality, sleep latency, sleep duration, habitual sleep efficiency, sleep disturbances, use of sleep medication, and daytime dysfunction. Each component score ranges from 0 to 3 , and a score $>1$ indicates problems in that aspect of sleep quality. The global score of sleep quality ranges from 0 to 21 with a higher score indicating poorer quality of sleep, whereas a cutoff score of $\leq 5$ indicates good sleep quality [16]. The original PSQI had received extensive support to confirm its good psychometric properties and high correlation with actual sleep $\log$ data $[17,18]$. Good reliability of the PSQI-C was also demonstrated among 793 Chinese adults, with a Cronbach's alpha $=0.84$ and a 2 -week test-retest reliability of $0.81[15]$.

\section{Ten-year risk of CVD}

Anthropometric measurements Anthropometric measurements were administered in the same manner for all participants. Body weight and height were measured without shoes. Body mass index (BMI) was calculated as weight divided by the square of height $\left(\mathrm{kg} / \mathrm{m}^{2}\right)$. Systolic blood pressure (SBP) and diastolic blood pressure (DBP) were measured by a mercury sphygmomanometer after at least $15 \mathrm{~min}$ of rest.

Framingham 10-year risk score The CVD risk in the participants was estimated by the office-based prediction model of the Framingham 10-year risk score (FRS) [19]. The predictors of the model include age, BMI, SBP, use of antihypertensive medication, medical history of diabetes, and current smoking status. This model revealed good performance in model discrimination and calibration, with a discrimination statistic of 0.785 [95\% confidence interval (CI), 0.764-0.806] and a calibration Chi-square of 10.24 [19]. The sensitivity and specificity of this model for CVD events in follow-up studies were 0.58 and 0.83 , respectively [19]. According to the American Heart Association guidelines for women' health, a FRS $\geq 10 \%$ is regarded as being at high risk for CVD [7].

Socio-demographic variables included age, marital status, education level, employment status, medical history, and drinking and smoking status, which were collected based on self-report information. The participants' physical exercise habit was evaluated by a single item: "How often do you do physical exercise?" A frequency of three times per week or above was regarded as a regular physical exercise habit [7]. 


\section{Statistical analysis}

Descriptive statistics were used to describe the sociodemographic characteristics, cardiovascular parameters, and sleep quality of the participants. The normality of each continuous variable was tested through skewness and kurtosis statistics.

Multivariate regression and logistic regression were used to determine the association between sleep quality, socio-demographic variables, and CVD risk. To identify potential predicting variables encoded in the regression models, bivariate analyses between participants' characteristics, sleep quality and FRS were conducted through independent t-test and Pearson correlation analyses as appropriate. As the FRS was calculated based on variables of age, gender, BMI, SBP, and smoking status [19], those variables were not examined in the bivariate analyses. A liberal significance level of 0.2 was applied to identify potential predictors, which would reduce the probability of excluding important variables from the model [20]. To reduce over-parameterization of the model, potential predictors with high co-variability $(r>0.7)$ and lowest correlation coefficient with the outcome variables were excluded from the model [20]. The two-sided level of significance was set at 0.05 . Statistical analysis was performed by the IBM SPSS version 21.0 [21].

\section{Results}

This study comprised of 154 Chinese post-menopausal women with a mean age of 63.65 (standard deviation, $\mathrm{SD}=4.47)$ years. Table 1 summarizes the sociodemographic characteristics and cardiovascular parameters of the post-menopausal women. A majority of participants were married $(81.8 \%)$ and had an education level of secondary or above (78.5\%). For those who were still in employment (10.4\%), most were employed as manual labourers, which required a high level of physically activity. The majority of the women $(72.1 \%)$ had regular physical exercise. Hypertension was the most prevalent chronic illness $(66,43 \%)$, followed by diabetes (13\%) and hypercholesterolemia (4\%). Among those with hypertension, only $25(37.9 \%)$ were taking antihypertensive medications. Only $1.3 \%$ of the participants were current smokers and 5.1\% were drinkers. Their mean BMI was $24.41(\mathrm{SD}=3.64) \mathrm{kg} / \mathrm{m}^{2}$, and $67.6 \%$ of the women were overweight or obese. The mean values for SBP and DBP were 126.20 (SD = 15.10) and 78.41 $(\mathrm{SD}=7.92) \mathrm{mmHg}$, respectively. The participants had a mean 10-year risk of CVD of $12.54 \%$, and half of them (51.3\%) were at high risk for CVD (FRS $\geq 10 \%$ ) [22, 23].

Findings from the PSQI showed that the sleep duration of the participants ranged from 1 to 9 (mean $=6.09$, $\mathrm{SD}=1.52$ ) hours, with $25.0 \%$ of the women having less than $5 \mathrm{~h}$ sleep. Time spent in bed ranged from 5 to 13
Table 1 Characteristics and cardiovascular parameters of the 154 post-menopausal women included in the analysis

\begin{tabular}{|c|c|c|}
\hline \multicolumn{2}{|c|}{ Socio-demographic characteristics } & \multirow{2}{*}{$\begin{array}{l}\text { Mean } \pm \text { SD or } \\
n(\%)\end{array}$} \\
\hline Age, years & (Range: 52-70) & \\
\hline \multirow[t]{2}{*}{ Marital status } & Married & $126(81.8 \%)$ \\
\hline & Widowed or divorced & $28(18.2 \%)$ \\
\hline \multirow[t]{3}{*}{ Educational level } & Primary or less ( $\leq 6$ years) & $33(21.4 \%)$ \\
\hline & Secondary ( $7-12$ years) & $104(67.5 \%)$ \\
\hline & Tertiary or above (> 12 years) & $17(11.0 \%)$ \\
\hline \multirow[t]{2}{*}{ Employment status } & Retired & $138(88.6 \%)$ \\
\hline & With full-time/part-time job & $16(10.4 \%)$ \\
\hline \multirow[t]{4}{*}{ Medical history } & Hypertension & $6642.9 \%)$ \\
\hline & $\begin{array}{l}\text {-Use of anti-hypertensive } \\
\text { medications }\end{array}$ & $25(37.9 \%)$ \\
\hline & Diabetes & $20(13.0 \%)$ \\
\hline & Hypercholesterolemia & $6(3.8 \%)$ \\
\hline With regular physical exercise & & $111(72.1 \%)$ \\
\hline Current smoker & & $2(1.3 \%)$ \\
\hline Current drinker & & $8(5.1 \%)$ \\
\hline \multicolumn{3}{|l|}{ Cardiovascular parameters } \\
\hline \multirow{3}{*}{$\begin{array}{l}\text { Body mass index (BMI), } \\
\mathrm{kg} / \mathrm{m}^{2}\end{array}$} & (Range: 16.9-36.73) & $24.41 \pm 3.64$ \\
\hline & Overweight (BMI: 23.0-24.9) & $40(26.0 \%)$ \\
\hline & Obese (BMI $\geq 25.0$ ) & $64(41.6 \%)$ \\
\hline $\begin{array}{l}\text { Systolic blood pressure, } \\
\mathrm{mmHg}\end{array}$ & (Range:90-180) & $126.20 \pm 15.10$ \\
\hline $\begin{array}{l}\text { Diastolic blood pressure, } \\
\mathrm{mmHg}\end{array}$ & (Range:59-100) & $78.41 \pm 7.92$ \\
\hline $\begin{array}{l}\text { Framingham 10-year risk score } \\
\text { (FRS), \% }\end{array}$ & (Range: 2.90-65.46) & $12.54 \pm 8.36$ \\
\hline High risk $F R S(\geq 10 \%)$ & & 79 (51.3\%) \\
\hline
\end{tabular}

(mean $=7.77, \mathrm{SD}=1.11)$ hours. The mean sleep efficiency, calculated by actual sleep duration / time spent in bed, was $78.35 \%$ (range: $7.69-100 \%$ ). Table 2 presents the participants' sleep quality, with regard to the seven component scores and the global score of PSQI. The global PSQI score was $8.58 \pm 4.37$. Six components, except for use of sleep medication, were scored $>1$. The component of sleep latency had the highest score (2.32), followed by sleep disturbance (1.51) and subjective sleep quality (1.26). Of the 154 participants, $136(88.3 \%)$ reported "had to get up to use the bathroom" at least once per week as the most common cause of sleep disturbance, followed by "cannot get to sleep within $30 \mathrm{mi}$ nutes" ( $n=110,71.4 \%)$, and "waking up in the middle of the night or early morning" ( $n=97,63.0 \%$ ).

In the multivariate regression, the FRS was analyzed as the dependent variable. Bi-variate analyses were 
Table 2 Sleep quality of the Chinese post-menopausal women $(N=154)$

\begin{tabular}{|c|c|c|}
\hline \multicolumn{2}{|l|}{ Sleep quality } & \multirow{2}{*}{$\begin{array}{l}\text { Mean } \pm \text { SD or } \\
n(\%) \\
1.26 \pm 0.91\end{array}$} \\
\hline Subjective sleep quality & (Range: 0-3) & \\
\hline Sleep latency & (Range: 0-3) & $2.32 \pm 0.69$ \\
\hline \multirow[t]{5}{*}{ Sleep duration } & (Range: $0-3$ ) & $1.15 \pm 1.11$ \\
\hline & $0(\geq 7$ h) & $58(37.7 \%)$ \\
\hline & $1(6-7$ h) & $41(26.6 \%)$ \\
\hline & $2(5-6 h)$ & $29(18.8 \%)$ \\
\hline & $3(<5 h)$ & $26(16.9 \%)$ \\
\hline Habitual sleep efficiency & (Range: 0-3) & $1.08 \pm 1.19$ \\
\hline Sleep disturbance & (Range: $0-3$ ) & $1.51 \pm 0.61$ \\
\hline Sleep medication & (Range: $0-3$ ) & $0.14 \pm 0.54$ \\
\hline Daytime dysfunction & (Range: 0-3) & $1.10 \pm 1.06$ \\
\hline Global PSQI score & (Range: 0-18) & $8.58 \pm 4.37$ \\
\hline Good sleep quality & Global PSQI $\leq 5$ & $42(27.3 \%)$ \\
\hline \multirow{3}{*}{$\begin{array}{l}\text { Global PSQI score among } \\
\text { groups of BP control }\end{array}$} & Hypertension_controlled $(n=52)$ & $9.77 \pm 3.93$ \\
\hline & Hypertension_uncontrolled $(n=14)$ & $8.36 \pm 3.56$ \\
\hline & Non_hypertension ( $n=88$ ) & $7.91 \pm 4.62$ \\
\hline \multicolumn{3}{|c|}{ Causes of sleep disturbances ( $\geq$ once per week) } \\
\hline \multicolumn{2}{|c|}{ Cannot get to sleep within $30 \mathrm{~min}$} & $110(71.4 \%)$ \\
\hline \multicolumn{2}{|c|}{ Waking up in the middle of the night or early morning } & $97(63.0 \%)$ \\
\hline \multicolumn{2}{|c|}{ Had to get up to use bathroom } & $136(88.3 \%)$ \\
\hline \multicolumn{2}{|c|}{ Cannot breathe comfortably } & $19(12.3 \%)$ \\
\hline \multicolumn{2}{|l|}{ Cough or snore loudly } & $70(45.5 \%)$ \\
\hline \multicolumn{2}{|l|}{ Feel too cold or hot } & $23(14.9 \%)$ \\
\hline \multicolumn{2}{|l|}{ Feel too cold or hot } & $48(31.2 \%)$ \\
\hline \multicolumn{2}{|l|}{ Having bad dreams } & $65(42.2 \%)$ \\
\hline \multicolumn{2}{|l|}{ Pain } & $61(39.6 \%)$ \\
\hline \multicolumn{2}{|l|}{ Other problems } & 57 (37.0\%) \\
\hline
\end{tabular}

PSQI Pittsburgh sleep quality index

${ }^{\mathrm{a}}$ Criteria for blood pressure (BP) control follows the recommendations of Eighth Joint National Committee (JNC 8 ) that for those $<60$ years, the control target is systolic BP (SBP) $<140 \mathrm{mmHg}$ and diastolic BP (DBP) $<90 \mathrm{mmHg}$; and for those $\geq 60$ years, the control target is $\mathrm{SBP}<150 \mathrm{mmHg}$ and DBP $<90 \mathrm{mmHg}$. The one way analysis of variance revealed: $\mathrm{F}=3.055, p=0.050054$

conducted to identify potential predicting variables of CVD risks. As presented in Table 3, five variables with a significance level $<0.2$ in the bi-variate analyses with FRS, including subjective sleep quality $(r=0.138, p=0.087)$, sleep duration $(r=0.106$, $p=0.191)$, sleep disturbance $(r=0.264, p=0.001)$, global PSQI score $(r=0.124, p=0.126)$, and employment status $(t=6.414, p=0.012)$. Given that global PSQI score was calculated based on all the seven component scores [15], to better understand the influences of specific sleep quality components on CVD risks, the variables of subjective sleep quality, sleep duration, and sleep disturbance, together with employment status
Table 3 Bivariate analyses between participants' characteristics, sleep quality and Framingham 10-year risk score $(n=154)$

\begin{tabular}{lll}
\hline Participants' characteristics & Statistical values & $p$-value \\
\hline Categorical variables $^{\mathrm{a}}$ & $t / \mathrm{F}$ & \\
Marital status & $t=0.076$ & 0.783 \\
Educational level & $\mathrm{F}=0.242$ & 0.786 \\
Employment status & $t=6.414$ & 0.012 \\
Physical exercise habit & $t=0.127$ & 0.722 \\
Current drinker & $t=1.561$ & 0.722 \\
Continuous variables & $r$ & \\
Subjective sleep quality & 0.138 & 0.087 \\
Sleep latency & 0.103 & 0.203 \\
Sleep duration & 0.106 & 0.191 \\
Habitual sleep efficiency & 0.077 & 0.343 \\
Sleep disturbance & 0.264 & 0.001 \\
Sleep medication & 0.006 & 0.936 \\
Daytime dysfunction & 0.021 & 0.800 \\
Global PSQl score & 0.124 & 0.126 \\
\hline
\end{tabular}

${ }^{a}$ Examined by independent $t$-test or one way analysis of variance

${ }^{\mathrm{b}}$ Examined by Person correlation analyses. PSQl: Pittsburgh sleep quality index

were included as independent variables in the regression model. Moreover, physical exercise habit was also adjusted in the regression model, for its significant influence on CVD risk observed in the previous study [11]. The multivariate regression model (Table 4) identified three variables that influenced the CVD risk: employment status $(p=0.016)$, sleep duration $(p=0.04)$, and sleep disturbance $(p<0.001)$.

Logistic regression analysis was also conducted to explore the relationship between sleep quality and CVD risk. The dependent variable is CVD risk, including a high-risk group (FRS $\geq 10 \%$ ) and a low-risk group (FRS $<10 \%)[22,23]$. As indicated in the bivariate analysis with CVD risk, five variables (subjective sleep quality, sleep duration, sleep disturbance, global PSQI score, and employment status) revealed $p$ values less than 0.2 . As the global PSQI score has considered the scores on

Table 4 Multivariate regression model for the cardiovascular disease risk of the Chinese post-menopausal women ${ }^{\text {a }}$

\begin{tabular}{lllll}
\hline Independent variables & $\beta$ & $95 \% \mathrm{Cl}$ & $\mathrm{SE}$ & $p$-value \\
\hline Constant & - & $(2.37,3.23)$ & 0.22 & $<0.001^{* * *}$ \\
Sleep duration & -0.18 & $(-0.32,-0.008)$ & 0.08 & $0.04^{*}$ \\
Sleep disturbance & 0.33 & $(0.27,0.85)$ & 0.14 & $<0.001^{* * *}$ \\
Employment status & -0.19 & $(-1.15,-0.12)$ & 0.26 & $0.016^{*}$ \\
Physical exercise habit & 0.02 & $(-0.31,0.39)$ & 0.18 & 0.83 \\
\hline
\end{tabular}

Adjusted $\mathrm{R}^{2}=0.110$

${ }^{a}$ The dependent variable was square rooted Framingham 10 year risk score $\mathrm{Cl}$ : confidence interval. SE: standard error. ${ }^{*} p<0.05 ;{ }^{* * *} p<0.001$ 
subjective sleep quality, sleep duration, and sleep disturbance. The independent variables in the logistic regress model included employment status and the sleep quality (including a good sleep quality group with PSQI $\leq 5$ and a poor sleep quality group with PSQI $>5$ ). Given the significant influence on CVD risk [11], physical exercise habit was also adjusted in the logistic regression model. The results demonstrated that women with good sleep quality were less likely to be at high risk for CVD, with an odds ratio (OR) of 0.51 (95\% CI: 0.26-0.97, $p=0.04)$.

\section{Discussion}

The Chinese post-menopausal women had poor sleep quality and increased CVD risks. The current study also identified a significant relationship between sleep quality and CVD risk, which provided valuable clues for developing interventions to improve the sleep quality and to reduce the CVD risks in post-menopausal women.

The socio-demographic characteristics of the study sample were consistent with those in previous studies among Chinese post-menopausal women [24, 25]. The mean FRS in the study sample was $12.54 \%$, indicating a high risk for CVD (> 10\%) among the Chinese postmenopausal women. Previous studies have also reported an increased CVD risk among post-menopausal women, which may be related to the reduced protective effects of estrogen on cardiovascular health $[8,26]$.

The participants reported a poor sleep quality, with a mean global PSQI score of $8.58(>6)$. Their sleep quality was much poorer than that of other age groups of Chinese women in other studies [13, 24]. Particularly, poor sleep quality had been consistently reported in previous studies among post-menopausal women [10, 25]. These findings suggest that poor sleep quality is common among post-menopausal women in both western and Chinese populations.

On average, the participants spent $7.77 \mathrm{~h}$ in bed per night, but only had $6.09 \mathrm{~h}$ of sleep. The more than 1-h of awake in bed reflected a severe problem with sleep latency (mean score $=2.32$ ) and may also have influenced their subjective perception of sleep quality [13]. Moreover, $71.4 \%$ of the participants reported "cannot get to sleep within 30 minutes" at least once per week. These findings are consistent with the high prevalence of insomnia reported in post-menopausal women in Asia [10, 24].

Most participants reported "have to get up to use bathroom," "cannot get to sleep within 30 minutes," and "waking up in the middle of the night or early morning." as the most common causes of sleep disturbance. These sleep problems may be due to the aging changes of their urinary system, or to menopausal symptoms such as hot flashes and night sweats [13,27]. Similar sleep problems were reported in the Wisconsin Sleep Cohort Study, in which post-menopausal women experienced more problems in initiating sleep $(\mathrm{OR}=2.77)$ and repeated episodes of waking up $(\mathrm{OR}=1.58)$ when compared with those of premenopausal women [27].

Sleep quality greatly affects the women's quality of life and role functioning in work places and family [28]. The poor sleep quality observed among Chinese postmenopausal women indicates the urgent need for interventions to improve their sleep quality, and in particularly to address the long sleep latency and severe sleep disturbances.

The present study also identified that among the postmenopausal women, there was a significant relationship between the CVD risks and sleep quality, particularly in sleep duration and sleep disturbance. Women who slept longer (with a lower component score in sleep duration) were at higher risks for CVD. Previous studies reported a U-shaped relationship between sleep duration and health, suggesting that too little or too much sleep correlates with adverse health outcomes [29]. A metaanalysis of 15 prospective studies also reported that when compared to participants with normal sleep duration, those with longer sleep duration ( $>8 \mathrm{~h}$ per night) had greater risks for total CVD incidents, CHD, and stroke [30]. The participants in the present study slept on average $6.09 \mathrm{~h}$ per night, and most $(81.8 \%)$ had normal sleep duration with $5-8 \mathrm{~h}$ of sleep per night [30]. The present study added evidence that among the postmenopausal women with normal sleep duration, longer sleep duration was associated with higher CVD risks.

The participants who experienced more sleep disturbances had higher CVD risks. However, the relationship between sleep disturbance and CVD risk has received limited attention in previous studies. The sleep disturbances as measured by the PSQI included "cannot get to sleep within 30 minutes," "waking up in the middle of the night or early morning," "had to get up to use bathroom," "cannot breathe comfortably," "cough or snore loudly," "feel too cold or hot," "having bad dreams," "pain," and other problems. All these reported sleep disturbances may reflect the severity of some menopausal symptoms, such as urinary frequency, anxiety, and hot flashes. Moreover, the latter symptoms were significantly associated with increased CVD risks $[5,6,31]$. In addition, these sleep disturbances may have interrupted the physiological recovery function of sleep and therefore led to poorer cardiovascular health [11]. Future studies should attempt to develop CVD prevention strategies by addressing the sleep disturbances experienced by post-menopausal women.

Interestingly, women who were still working were at lower risk for CVD $(\beta=-0.19, p=0.016)$. Some women might stop working due to the comorbidity of 
hypertension, diabetes, or poor sleep quality. These health problems would contribute to an increased CVD risk. On the other hand, the majority of the women in the working group were manual labourers, who are likely to have higher levels of physical activity compared with those in the non-working group. Moreover, the working women were significantly younger than the non-working women (57.8 vs. 64.3 years, $p<0.001$ ). Because age is a significant predictor in the algorithm of the FRS [19], the correlation between working status and CVD risk should be interpreted with caution. Future studies should pay attention to promote the cardiovascular health among post-menopausal women who are not working, such as improving their physical activity levels.

The CVD risk was found to be reduced by half in women who had better sleep quality than those who had poor sleep quality $(\mathrm{OR}=0.51, p=0.04)$. This finding was consistent with the results of previous studies. Good sleep quality has been recognized as a beneficial factor for cardiovascular health, and may prevent the development of CVD [9]. One study in the Netherlands reported that short-duration sleepers with poor sleep quality had a $63 \%$ increased risk for CVD, and a $79 \%$ higher risk for CHD when compared to normal-duration sleepers who had good sleep quality [11]. This evidence further strengthens the need to include sleep quality as an important indicator when assessing CVD risk among Chinese post-menopausal women.

Considering the critical contribution of SBP and use of anti-hypertensive medication on CVD risks and the close relationship between sleep quality and CVD risks in the current study participants, further analysis was conducted to explore the relationship between sleep quality and BP control. The Eighth Joint National Committee recommended that for those $<60$ years, the control target is SBP $<140 \mathrm{mmHg}$ and DBP $<90 \mathrm{mmHg}$; and for those $\geq 60$ years, the control target is $\mathrm{SBP}<150 \mathrm{mmHg}$ and DBP $<90 \mathrm{mmHg}$ [32]. Accordingly, the participants were divided into three groups: those with hypertension and controlled BP; those with hypertension but uncontrolled BP; and a group without hypertension. However, the one way analysis of variance did not revealed statistically significant difference in sleep quality among the groups $(\mathrm{F}=3.055, p=0.050054)$.

To the best of our knowledge, this is the first study to examine the relationship between sleep quality and CVD risk among Chinese post-menopausal women. Nonetheless, this study had some limitations. First, this was a cross-sectional study with an internal limitation in confirming the cause-and-effect relationships. Future study should use a prospective study design with long-term follow-up to further explore the relationship between sleep quality and CVD risk. Secondly, sleep quality was measured by the self-reported PSQI. Previous studies had extensively used such a self-report system, even through a single item to assess sleep duration or sleep quality. Anyway, the PSQI is a comprehensive assessment of sleep quality and has revealed good performance in psychometric properties [27, 30]. To eliminate the possibility of self-reporting bias, future studies should employ a more objective approach to measure sleep quality, such as polysomnography and actigraphy. Moreover, the medical history of hypertension, diabetes, and hypercholesterolemia was also collected based on participants' self-report information. To get more accurate information, future studies could conduct blood tests or retrieve data from participants' medical records.

\section{Conclusions}

In conclusion, this study identified that Chinese postmenopausal women had poor sleep quality and increased CVD risk. Special attention should be paid to improve the sleep quality and cardiovascular health in these women. As indicted by the significant relationship between sleep quality and CVD risk, poor sleep quality might increase the CVD risk in post-menopausal women. To improve their cardiovascular health, future interventions should target towards strategies for improving sleep quality and for addressing sleep disturbances.

\section{Abbreviations \\ BMI: Body mass index; CHD: Coronary heart disease; Cl: Confidence interval; CVD: Cardiovascular disease; DBP: Diastolic blood pressure; FRS: Framingham 10-year risk score; OR: Odds ratio; PSQI: Pittsburgh sleep quality index; SBP: Systolic blood pressure; SD: Standard deviation}

\section{Acknowledgements}

The authors would like to thank all the participants of the study.

Funding

Nil.

Availability of the data and materials

Data were available upon request from the corresponding author.

\section{Authors' contributions}

SYC, QW, and XML were involved in the study design and coordination. QW and XML conducted the data collection. QW, HYC and SWL completed the data analysis. SYC, QW, HYC and SWL drafted the manuscript. XML, EMW and JWS revised the manuscript for quality, consistency and accuracy. All authors have read and approved the final manuscript.

\section{Ethics approval and consent to participate}

The study was approved by the Ethical Committee of the Xian Jiaotong University [13]. Each participant received detailed explanation about the study and provided written informed consent before participating in the study.

\section{Consent for publication}

Not applicable.

\section{Competing interests}

The authors declare that they have no competing interests.

\section{Publisher's Note}

Springer Nature remains neutral with regard to jurisdictional claims in published maps and institutional affiliations. 


\section{Author details}

'The Nethersole School of Nursing, The Chinese University of Hong Kong, Esther Lee Building, The Chinese University of Hong Kong, Shatin, New Territories, Hong Kong. ${ }^{2}$ The Faculty of Nursing, College of Medicine, Xian Jiaotong University, Xi'an, Shaanxi Province, China.

\section{Received: 14 January 2016 Accepted: 28 August 2017}

\section{Published online: 11 September 2017}

\section{References}

1. Feng JJ. Worries about the sexual health of Chinese women during menopause, Implies from the Euro-Asia study on health of menopause women. Beijing: People's Daily; 2006. Vol. 14

2. Shifren JL, Gass ML. NAMS recommendations for clinical Care of Midlife Women Working Group: the North American Menopause Society recommendations for clinical care of midlife women. Menopause. 2014;21(10):1038-62.

3. He L, Tang X, Li N, Wu Y, Wang J, Li J, Zhang Z, Dou H, Liu J, Yu L. Menopause with cardiovascular disease and its risk factors among rural Chinese women in Beijing: a population-based study. Maturitas. 2012;72(2):132-8.

4. Colditz GA, Willett WC, Stampfer MJ, Rosner B, Speizer FE, Hennekens CH. Menopause and the risk of coronary heart disease in women. N Engl J Med. 1987:316(18):1105-10.

5. Van der Schouw Y, van der Graaf Y, Steyerberg E, Eijkemans M, Banga J. Age at menopause as a risk factor for cardiovascular mortality. Lancet. 1996;347(9003):714-8.

6. Rosano G, Vitale C, Marazzi G, Volterrani M. Menopause and cardiovascular disease: the evidence. Climacteric. 2007;10(sup1):19-24.

7. Mosca L, Benjamin EJ, Berra K, Bezanson JL, Dolor RJ, Lloyd-Jones DM, Newby LK, Pina IL, Roger VL, Shaw LJ. Effectiveness-based guidelines for the prevention of cardiovascular disease in women-2011 update: a guideline from the American Heart Association. J Am Coll Cardiol. 2011;57(12):1404-23.

8. Polo-Kantola P, Saaresranta T, Polo O. Aetiology and treatment of sleep disturbances during perimenopause and postmenopause. CNS drugs. 2001;15(6):445-52

9. Chung KF, Tang MK. Subjective sleep disturbance and its correlates in middle-aged Hong Kong Chinese women. Maturitas. 2006:53(4):396-404.

10. Huang K, Xu L, Nasri N, Jaisamrarn U. The Asian menopause survey: knowledge, perceptions, hormone treatment and sexual function. Maturitas. 2010;65(3):276-83.

11. Hoevenaar-Blom MP, Spijkerman AM, Kromhout D, van den Berg JF, Verschuren WM. Sleep duration and sleep quality in relation to 12-year cardiovascular disease incidence: the MORGEN study. Sleep. 2011:34(11):1487-92.

12. Shahar E, Whitney CW, REdline S, Lee ET, Newman AB, Javier Nieto F, O'Connor GT, Boland LL, Schwartz JE, Samet JM. Sleep-disordered breathing and cardiovascular disease: cross-sectional results of the sleep heart health study. Am J Respir Crit Care Med. 2001;163(1):19-25.

13. Wang Q, Li X. Study on sleep quality of elderly in communities in Xi'an city and its influencing factors. Chin Nurs Res. 2012;7:591-4.

14. Harlow SD, Gass M, Hall JE, Lobo R, Maki P, Rebar RW, Sherman S, Sluss PM, De Villiers TJ. Executive summary of the stages of reproductive aging workshop 10: addressing the unfinished agenda of staging reproductive aging. Climacteric. 2012;15(2):105-14.

15. Liu X, Tang M, Hu L. Reliability and validity of the Pittsburgh sleep quality index. Chinese Journal of Psychiatry. 1996;29:103-7.

16. Buysse DJ, Reynolds CF, Monk TH, Berman SR, Kupfer DJ. The Pittsburgh sleep quality index: a new instrument for psychiatric practice and research. Psychiatry Res. 1989;28(2):193-213.

17. Backhaus J, Junghanns K, Broocks A, Riemann D, Hohagen F. Test-retest reliability and validity of the Pittsburgh sleep quality index in primary insomnia. J Psychosom Res. 2002;53(3):737-40.

18. Mollayeva T, Thurairajah P, Burton K, Mollayeva S, Shapiro C, Colantonio A. The Pittsburgh sleep quality index as a screening tool for sleep dysfunction in clinical and non-clinical samples: a systematic review and meta-analysis. Sleep Med Rev. 2015;25:52-73.

19. D'Agostino RBS, Vasan RS, Pencina MJ, Wolf PA, Cobain M, Massaro JM, Kannel WB. General cardiovascular risk profile for use in primary care: the Framingham heart study. Circulation. 2008;117(6):743-53.
20. Tabachnick BG. In: Tabachnick BG, Fidel LS, editors. Using multivariate statistics /. 6th ed. Boston: Boston: Pearson Education; 2013.

21. Corp I: IBM SPSS statistics for Windows, version 21.0. 2012.

22. Lloyd L, Langley-Evans SC, McMullen S. Childhood obesity and risk of the adult metabolic syndrome: a systematic review. Int J Obes. 2012;36(1):1-11.

23. Grundy SM, Cleeman II, Merz CNB, Brewer HB, Clark LT, Hunninghake DB, Pasternak RC, Smith SC, Stone NJ. Implications of recent clinical trials for the national cholesterol education program adult treatment panel III guidelines. J Am Coll Cardiol. 2004:44(3):720-32.

24. Chen H, Liu J, Zhang X. Association between sleep quality and psychosomatic symptoms in menopause women in community. Medical Journal of Chinese People's Health. 2008;20(3):200-3.

25. Lin Y, Li F, Zhang J, Wang L. Investigation and analysis on health status in climacteric women of Xi'an City. Journal of Xi'an Jiaotong University (Medical Sciences). 2008;1:110-4.

26. Kannel WB, Hjortland MC, McNamara PM, Gordon T. Menopause and risk of cardiovascular disease: the Framingham study. Ann Intern Med. 1976;85(4):447-52

27. Young T, Rabago D, Zgierska A, Austin D, Laurel F. Objective and subjective sleep quality in premenopausal, perimenopausal, and postmenopausal women in the Wisconsin sleep cohort study. Sleep. 2003;26(6):667-72.

28. Lee KA, Kryger MH. Women and sleep. J Women's Health. 2008;17(7):1189-90.

29. Knutson KL, Turek FW. The U-shaped association between sleep and health: the 2 peaks do not mean the same thing. Sleep. 2006:29(7):878-9.

30. Cappuccio FP, Cooper D, D'Elia L, Strazzullo P, Miller MA. Sleep duration predicts cardiovascular outcomes: a systematic review and meta-analysis of prospective studies. Eur Heart J. 2011;32(12):1484-92.

31. Szmuilowicz ED, Manson JE, Rossouw JE, Howard BV, Margolis KL, Greep NC, Brzyski RG, Stefanick ML, O'Sullivan MJ, Wu C, Allison M, Grobbee DE, Johnson KC, Ockene JK, Rodriguez BL, Sarto GE, Vitolins MZ, Seely EW. Vasomotor symptoms and cardiovascular events in postmenopausal women. Menopause. 2011;18(6):603-10

32. James PA, Oparil S, Carter BL, Cushman WC, Dennison-Himmelfarb C, Handler J, Lackland DT, LeFevre ML, MacKenzie TD, Ogedegbe O, Smith SC. 2014 evidence-based guideline for the management of high blood pressure in adults: report from the panel members appointed to the eighth joint National Committee (JNC 8). JAMA. 2014:311(5):507-20.

\section{Submit your next manuscript to BioMed Central and we will help you at every step:}

- We accept pre-submission inquiries

- Our selector tool helps you to find the most relevant journal

- We provide round the clock customer support

- Convenient online submission

- Thorough peer review

- Inclusion in PubMed and all major indexing services

- Maximum visibility for your research

Submit your manuscript at www.biomedcentral.com/submit
C) BioMed Central 